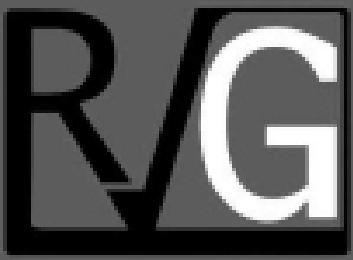

Año 24 No. 88

Octubre - Diciembre 2019

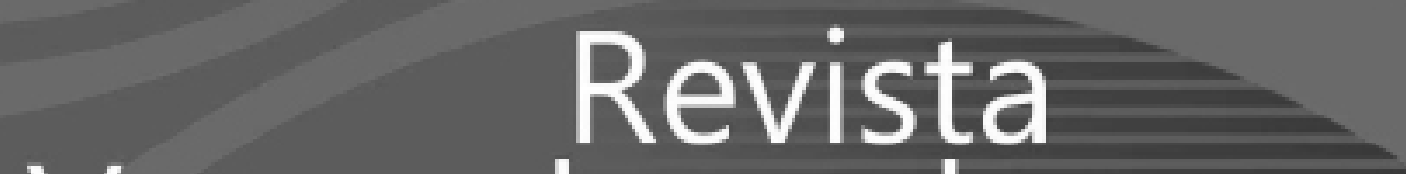

Venezolana de

verencla

UNIVERSIDAD DEL ZULIA (LUZ)

Facultad de Ciencias Económicas y Sociales

Centro de Estudios de la Empresa 


\title{
Gestión del diseño curricular universitario en Ecuador
}

\author{
Oscar Santiago, Barzaga Sablón ${ }^{1}$ \\ Carlos Enrique, Santos Loor ${ }^{2}$ \\ Oscar Elías, Bolívar Chávez ${ }^{3}$ \\ Johanna María, Zambrano Sornoza ${ }^{4}$
}

\section{Resumen}

El presente trabajo aborda la gestión del diseño curricular universitario en Ecuador, particularmente se aborda desde la perspectiva multicultural, intercultural y transcultural la carrera de Licenciatura en Educación Básica y se toma como referente el valor que tiene la formación profesional del estudiante con el desarrollo de las ciencias sociales, bajo un enfoque de la multiculturalidad que actualmente se adelanta en Ecuador. La metodología consistió en realizar un estudio descriptivo de campo y como instrumento de recolección de información se aplicó entrevistas semiestandarizadas. Los resultados revelan: a) necesidad del desarrollo de competencias multiculturales, desde la carrera de educación básica, lo que se pone de manifiesto en la formación de un profesional altamente competente capaz de interactuar en los diferentes escenarios multiculturales; b) el rediseño curricular orientado hacia las asignaturas de historia, filosofía, etnología, etnografía, antropología, sociología entre otras, aplicadas a la enseñanza de este

Recibido: 23-01-19

Aceptado: 23-03-19

Doctor en Ciencias, PhD. Profesor de la Facultad de Filosofía, Letras y Ciencias de la Educación, de la Universidad Técnica de Manabí. ORCID: 0000-0002-5303-949x.

2 Doctor en Ciencias de la Educación. Profesor de la Facultad de Filosofía, Letras y Ciencias de la Educación, de la Universidad Técnica de Manabí. Email: carlossantosloor@hotmail.comORCID: 0000-0001-7187-6163.

3 Magister en Nuevas Tecnologías Aplicadas a la Educación. Profesor de la Facultad de Filosofía, Letras y Ciencias de la Educación, de la Universidad Técnica de Manabí.Email osckarellyrecords@hotmail.comORCID:000-0002-0910-352x

4 Magister en Gerencia Educativa. Profesor de la Facultad de Filosofía, Letras y Ciencias de la Educación, de la Universidad Técnica de Manabí.Emailjohazs@hotmail.com.ORCID:0000-0001-7264-1374. 
fenómeno de la multiculturalidad en la formación profesional del estudiante de Licenciatura en Educación Básica. Se concluye que el proceso de gestión del rediseño curricular pasa por una diversidad de etapas que demanda la necesidad de un rediseño curricular que desarrolle competencias vinculadas a la multiculturalidad.

Palabras clave: Competencia profesional; interculturalidad; multiculturalidad; rediseño curricular; transculturalidad.

\title{
Management of university curriculum design in Ecuador
}

\begin{abstract}
The present work deals with the management of university curricular design in Ecuador, particularly from a multicultural, intercultural and cross-cultural perspective, the Bachelor's Degree in Basic Education is taken and the value of the student's professional training with the development of students is taken as a reference social sciences, under a multicultural approach that is currently being carried out in Ecuador. The methodology consisted of conducting a descriptive field study and as a tool for collecting information semi-standardized interviews were applied. The results reveal: a) The need for the development of multicultural competences, from the Basic Education degree, which is evident in the formation of a highly competent professional capable of interacting in the different multicultural scenarios; b) The curricular redesign oriented towards the subjects of history, philosophy, ethnology, ethnography, anthropology, sociology among others, applied to the teaching of this phenomenon of multiculturalism in the professional training of the Bachelor of Basic Education student. It is concluded that the curriculum redesign management process goes through a diversity of stages that demands the need for a curriculum redesign that develops competences linked to multiculturalism.
\end{abstract}

Keywords: Professional competence; interculturality; multiculturalism; curriculum redesign; transculturality

\section{Introducción}

El análisis de las competencias desde la multiculturalidad se utilizaron métodos teóricos tales como: la abstracción científica, este método se aplicó a través de las operaciones lógicas del pensamiento: análisis síntesis e inducción- deducción. Esto permitió la descomposición de los rasgos fundamentales de las competencias 
multiculturales en el área de la Enseñanza Básicas, para luego integrar mentalmente las partes analizadas y establecer sus relaciones esenciales en calidad de elementos que forman parte del núcleo teórico del objeto de estudio. La inducción-deducción permitió definir una serie de interrogantes en torno a la teoría relacionada con el tema de la multiculturalidad en la mencionada carrera objeto de estudio (Nocedo:1983).

El empleo del método teórico histórico-lógico permitió valorar los diferentes aportes de diversos autores y tendencias que conforman la teoría entorno a la multiculturalidad, para establecer las tendencias y perspectivas de su desarrollo histórico (Nocedo:1983).

El método comparativo permitió comparar las diversas teorías desde la perspectiva multidisciplinaria acerca de la multiculturalidad en la Enseñanza Básica a nivel internacional, nacional y local, con el objetivo de definir los elementos comunes y diferenciales de la mencionada actividad (Nocedo: 1983). El empleo de la modelación científica, develó el contenido y las formas de las manifestaciones multiculturales, así como los indicadores básicos para medirla (Nocedo:1983).

El método cualitativo historia de vida (Olabuénaga: 1999), favoreció acceder a importante información como: la historia de vida de la carrera y los graduados, las limitaciones en el desarrollo las competencias, perspectivas y tendencias de su desarrollo. Se implementó a partir de una guía de temas, a través de ella va introduciendo nuevas interrogantes que satisfacen las necesidades de información del tema seleccionado.

El método análisis de documentos, permitió obtener información referente a los documentos referentes a la carrera y los graduados: informes, resultados docentes, oficios, convenios, proyectos, entre otros. A los documentos se les aplicó el procedimiento siguiente: el análisis de dominio, que abarcó los aspectos descriptivos del documento, el análisis de taxonomías, que favoreció profundizar en los aspectos estructurales, el análisis de componentes principales, y el análisis de temas que establecer los aspectos estructurales de los documentos referentes a los temas abordados relacionados.

Para el análisis estadístico y pruebas de hipótesis se utilizó IBM. SPSS. Statistics versión 22, en español.

Esta investigación se centra en la carrera de Licenciatura en Educación Básica fundada en la Facultad de Filosofía, Letras y Ciencias de la Educación de la Universidad Técnica de Manabí, mediante informes aprobados por el consejo académico permanente del Consejo Universitario. La carrera juega un importante papel en la solución de los problemas del sector que tiene como rasgo fundamental la multiculturalidad, lo que exige una concepción creativa e innovadora en la solución de sus problemas desde la multiculturalidad.

\section{Interculturalidad: Algunas nociones básicas para reflexionar}

El concepto de interculturalidad aborda la interacción entre dos o más culturas desde la horizontalidad y la sinergia. Esto implica una relación equilibrada entre las culturas donde ninguna es superior a otra, lo que permite la integración y la convivencia armónica de todos los individuos. Estas relaciones interculturales se caracterizan por el respeto hacia la diversidad, aunque, en 
ocasiones, se evidencien conflictos que se resuelven a través del respeto, el diálogo y la concertación.

Recientemente se comienza con la discusión del concepto de interculturalidad, desde la literatura sobre la comunicación, la antropología, la sociología y la educación a diferencia del concepto de multiculturalismo y del pluralismo por su rasgo esencial de promover el diálogo y el acercamiento entre culturas.

La interculturalidad depende de múltiples factores como: las diferentes acepciones de cultura, las limitaciones comunicativas, la ausencia de políticas estatales y las diferencias económicas (Castillo y Guido: 2015). Desde la ética podemos descubrir que la forma en que la interculturalidad se involucra en el enraizamiento de los valores sociales a través del respeto a la diversidad, donde cada individuo tiene derecho a manifestar libremente su ser. La ética inculca valores para construir sociedades democráticas, integradas y donde la armonía sea factor fundamental de la interacción social.

La interculturalidad y la integración son factores inseparables, donde las posibilidades de desarrollo son variadas, permitiendo entrar en contacto con culturas diferentes sin contradecir la propia identidad, enriqueciendo ampliamente las culturas interactuantes Diez y Mateo, 2011.

Existe una amplia diversidad de formas de promover la interculturalidad en la sociedad. El primer lugar lo ocupa la familia, donde los niños deben educarse libremente, sin imponerles juicios preconcebidos, dogmas 0 esquemas rígidos, sino en desarrollar la habilidad de pensar y ser tolerante a lo que es diferente Clouet, 2013. Otro lugar importante lo ocupa la implementación de proyectos que permitan eliminar de forma progresiva los prejuicios en torno a individuos o grupos. En último lugar se sitúa, la integración que se promueve desde los más altos cargos, permitiendo que cualquier ciudadano tenga los mismos derechos sin limitarles por: sus capacidades, ideologías, color de la piel, género, edad, etnia o lugar de origen.

El análisis intercultural consta de tres momentos: la negociación, que es la simbiosis que se requiere para lograr la comprensión y evitar la confrontación; la penetración, que implica salir del propio lugar para tomar el punto de vista del otro y la descentralización, que es una perspectiva de reflexión. Es importante destacar que para el logro de una interculturalidad efectiva es necesario que se cumplan tres condiciones básicas como: la visión dinámica de las culturas, el establecimiento de vínculos por medio de la comunicación y la conformación de sistema que garantice la igualdad de derechos entre todos.

\section{Contexto de la formación del estudiante adscritos a la carrera en Licenciatura en Educación Básica en América Latina}

en Educación Básica, en el contexto intercultural, proporciona a los estudiantes la oportunidad de desarrollar los conocimientos $y$ habilidades necesarias para abordar la especialidad a partir de la intervención en las instituciones educativas desde la diversidad de culturas en lo nacional e internacional. La educación rebasa las fronteras nacionales para adentrarse en los marcos de una economía globalizada. Lo que implica el empleo de nuevas 
estrategias educativas, que incluya en la investigación la multiculturalidad e interculturalidad.

Lo anteriormente expuesto, indica el empleo de nuevos enfoques integrales bajo la agenda 2030 de la Unesco, referida a una educación inclusiva, equitativa y de calidad en la teoría y en la práctica, para enfrentar los nuevos desafíos que este contexto impone en la preparación de los estudiantes para una carrera en el entorno educativo global. Los mismos adquirirán los conocimientos y habilidades necesarias para un desempeño exitoso en el mundo intercultural, ganando tanto en el desarrollo de un pensamiento crítico, como en el dominio de habilidades analíticas cruciales para la toma de decisiones en un mundo multicultural en plena evolución.

El enfoque intercultural incluye aspectos conceptuales, teóricos y prácticos relevantes de la carrera. Al graduarse, los licenciados en educación, en un contexto intercultural, estarán preparados para un desempeño exitoso en el campo de la enseñanza y la gestión educativa trabajando para los diferentes niveles de enseñanza, los departamentos gubernamentales, organizaciones e instituciones (Orozco, 2014: 45).

El análisis del desarrollo de competencias unida a la multiculturalidad en la carrera de Educación Básica, es un estudio teórico, donde se realiza un análisis crítico, a partir del aporte de importantes investigadores sobre el tema. Este enfoque científico, exige métodos que corresponden con los métodos teóricos de investigación y del paradigma cualitativo, que en la metodología de la investigación se conoce bajo el concepto de triangulación de métodos Nocedo, 1983.

Entorno a la concepción multicultural, intercultural y transcultural en la formación del estudiante de la carrera en Licenciatura en Educación Básica. Es necesario señalar que el enfoque multicultural de la formación profesional de la carrera de Educación Básica General, es un tema poco abordado en la literatura científica, por no decir, prácticamente virgen, es por ello que no se encuentran suficientes referentes en las bases de datos, bibliotecas y publicaciones periódicas sometidas a revisión. Esto lo convierte en un objeto de investigación de mercado interés en el ámbito de las ciencias de la educación.

La educación, abordada desde la multiculturalidad, exige una nueva generación de docentes que interpreten lo complejo del mundo en que nos desenvolvemos Cruz, 2013. Es por eso que las instituciones educativas que operan en el Ecuador demandan jóvenes altamente calificados y con capacidad de lograr los mejores resultados locales y globales.

El carácter dinámico de la ciencia pedagógica obliga a establecer un diseño curricular abierto, flexible, dinámico, capaz de integrar nuevos conocimientos en un sistema único y coherente, en base al desarrollo de dos ciclos: uno de Formación Básica y otro de Formación Profesional, incluyendo un espacio de asignaturas opcionales para estimular al alumno a profundizar en temas desde la multiculturalidad que a lo largo de la carrera han despertado su interés.

En la mayoría de los programas curriculares, en el ciclo básico tiene por objetivos: incorporar el conocimiento universal y abstracto de los ejes temáticos propuestos en la carrera, terminología específica, generalizaciones, teorías, identificar y valorar lo aprendido; establecer relaciones causales, consecuencias de hechos, definir tendencias y secuencias, comparaciones, interpretación y descripciones, desde una perspectiva multidisciplinaria y multicultural; aplicar 
lo aprendido a la solución de problemas y a situaciones nuevas de carácter complejo, organizacional, de contextos individuales de toma de decisión, estimular la creatividad, la formación independencia cognoscitiva y el deseo de progreso.

Las políticas públicas en materia de educación diseñadas e implementadas en Ecuador, a través de la matriz de tensiones y problemas de la zona cuatro (GAD Cantón Sucre, 2012) determina los contextos multiculturales y ejes que deben ser atendidos estratégicamente con el fin de mejorar la calidad de vida de los ciudadanos. La carrera se articula al Plan de Desarrollo Nacional del cantón Portoviejo 2018, es necesario señalar que el enfoque multicultural de la formación profesional de la carrera de educación es un tema poco abordado en la literatura científica, por no decir, prácticamente virgen, por ello no se encuentran referentes en las bases de datos, bibliotecas y publicaciones periódicas sometidas a revisión. Esto lo convierte en un objeto de investigación de marcado interés en el ámbito de las ciencias de la educación.

\section{Gestión del diseño curricular de la llicenciatura en Educación Básica fundada en la Facultad de Filosofía, Letras y Ciencias de la Educación de la Universidad Técnica de Manabí}

\footnotetext{
Para caracterizar a los graduados de la carrera de Licenciatura en Educación Básica, en relación a su formación desde la multiculturalidad, la interculturalidad y la transculturalidad, se realizó un diagnóstico. El diagnóstico
}

se realizó a una muestra aleatoria de 122 graduados, entre los años 2016 y 2017, de la carrera de Licenciatura en Educación Básica, de una población de 350. El tamaño de muestra se calculó a través de la fórmula: $n=$ $\mathrm{Z}^{2 *} \mathrm{p}^{*} \mathrm{q}^{*} \mathrm{~N} / \mathrm{e}^{2 *}(\mathrm{~N}-1)+\mathrm{Z}^{2 *} \mathrm{p}^{*} \mathrm{q}$, donde $\mathrm{Z}$ es la distribución normal debajo de la curva, (p) es la probabilidad de éxito y (q) es la probabilidad de no éxito y (e) es el error, se calculó para una probabilidad de éxito del $95 \%$ y un error del $5 \%$. Obteniéndose, $\mathrm{n}=\quad 4^{2 *} 50 * 50 * 350 \quad 125^{2 *}(350-1)$ $+4^{2 *} 50 * 50=3500000 / 18725=187$, a aplicar la fórmula para corregir el tamaño de la muestra obtenido de 187 , nc (tamaño de muestra corregido), nc= $\mathrm{n} / 1+(\mathrm{n} / \mathrm{N})=187 / 1,53=122$, por lo que el tamaño de la muestra es 122 graduados.

Con respecto al desarrollo de competencias vinculada a la multiculturalidad, como resultado de la formación general proporcionada a los graduados en la Universidad Técnica de Manabí (UTM), se tuvieron en cuenta las competencias de: relacionar la cultura de origen con otras culturas; establecer contacto con diferentes culturas; de mediador entre su cultura y otras culturas; asimilar aspectos culturales de diferentes culturas, comunicación con otras culturas, tolerar las influencias de culturas diferentes, conocer su cultura y las demás culturas y superar los tabúes que limitan la interacción cultural.

La encuesta aplicada evidenció que de los 122 entrevistados, más del $50 \%$, 6 de cada 10 encuestados, en las variables antes mencionadas, 68 el $55 \%$ evalúa las competencias vinculadas con la interculturalidad entre poco competente y muy no competente, el resto, 4 de cada 10 encuestados, 54 el $45 \%$, las calificó entre competente y muy competente.

Para poder determinar sí la 
diferencia entre la proporción de las personas entrevistadas es significativa se realizó la prueba de hipótesis de proporciones, donde la hipótesis de nulidad Ho, establece que no hay diferencias significativas entre ambas proporciones $\mathrm{p} 1=\mathrm{p} 2$, y la alternativa $\mathrm{Hi}$ : $\mathrm{p} 1 \neq \mathrm{p} 2$, considera que sí existe diferencia significativa entre ambas proporciones, tomando como nivel de significación el 0,05 .

El pv ( $p$ valor) obtenido es 0 $0,075193>$ que el nivel de significación $\alpha=0,05$, por lo que se rechaza la prueba de hipótesis de igualdad de proporciones. Se puede afirmar, entonces, que existe una diferencia significativa entre la proporción de entrevistados que afirman que existen bajos niveles de competencia vinculadas con la multiculturalidad y los que consideran que existe un nivel alto y aceptable de competencias vinculadas con la multiculturalidad, predominando los que afirman que los niveles de competencia de los graduados en relación a la multiculturalidad es bajo.

En relación, al desarrollo de competencias unidas a la multiculturalidad en la carrera, de los 122 graduados, 67 el $55 \%$, consideran que en la misma no se desarrollan en los estudiantes estas competencias, solo 55 , el $45 \%$, afirman que sí se desarrollan competencias vinculadas con la multiculturalidad.

Para establecer sí existe diferencia significativa entre la proporción de los graduados entrevistados, que afirman que en la carrera sí se desarrollan las competencias relacionadas con la multiculturalidad y los que afirman que el nivel de desarrollo de dichas competencias es bajo. Se realizó la prueba de hipótesis de comparación de proporciones, donde la hipótesis de nulidad Ho, establece que no hay diferencias significativas entre ambas proporciones $\mathrm{p} 1=\mathrm{p} 2$, y la alternativa $\mathrm{Hi}: \mathrm{p} 1 \neq \mathrm{p} 2$, considera que sí existen diferencias significativas, tomando como nivel de significación el 0,05.

El pv ( $p$ valor) obtenido es 0,127347 $>$ que el nivel de significación $\alpha=0,05$, por lo que se rechaza la prueba de hipótesis de igualdad de proporciones. Se puede afirmar, entonces, que existe una diferencia significativa entre la proporción de entrevistados que afirman que existen bajos niveles de competencia vinculadas con la multiculturalidad en la carrera y los que consideran que existe un nivel alto y aceptable de competencias vinculadas con la multiculturalidad, predominando los que afirman que los niveles de competencia en los estudiantes de la carrera en relación a la multiculturalidad es bajo.

Al estudiar la malla curricular se pone de relieve que asignaturas tales como: la sociología, la antropología, la etnología, la etnografía y la geografía aplicada a la enseñanza y que favorecen el desarrollo de competencias en la multiculturalidad, no están presentes. Estas asignaturas son claves para el desarrollo de un pensamiento y una praxis intercultural, pues en su objeto de estudio, ellas, en su conjunto, ofrecen una visión, información y conocimiento de las diferentes culturas tanto en el ámbito nacional como internacional.

La etnografía, la antropología, la etnología y la sociología (sociología de la cultura), ofrecen valiosa información sobre la identidad y diversidad cultural de los pueblos. La identidad ecuatoriana tiene que ver con nuestra historia individual y la historia colectiva, con nuestro pasado ancestral y las tradiciones culturales que son partes inseparables de Ecuador, las que constituyen un reflejo de las relaciones entre las personas, 
comunidades y etnias (Guber, 2001).

$$
\text { Las asignaturas antes }
$$

mencionadas abordan los rasgos tanto identitarios como diferenciales de las diferentes culturas, conocimiento valioso para el conocimiento, la toma de decisiones en lo referente a los diversos tipos de enseñanza donde accionar, el desconocimiento de las diferentes culturas y sus formas comerciales es de capital importancia para un buen desempeño en la esfera de la enseñanza. $\mathrm{Su}$ desconocimiento implicaría tomar decisiones no adecuadas y trazarse estrategias poco eficaces en lo que a la enseñanza se refiere.

$\begin{array}{ccc}\text { Lo } & \text { ecuatoriano, } & \text { adquiere } \\ \text { dimensiones } & \text { simbólicas en un }\end{array}$ proceso de transculturación, redefine el concepto de ecuatoriano y su identidad y reincorporan la tradición cultural con el factor de identidad colectiva, incorporando lo andino, lo afro ecuatoriano, sus tradiciones religiosas desde la colonia, la gastronomía como símbolo de la ecuatorianidad colectiva, la tradición africana, la tradición chinacantonesa, como la tradición española y occidental.

La revalorización de las tradiciones culturales retroalimenta la construcción de ecuatorianidad como identidad en la carrera. Esto se revela claramente en los estereotipos: indio, blanco, negro, mestizo, cholo y montubio, propios de la sociedad ecuatoriana que se diluyen y lo mestizo se convierte en realmente en una forma cultural. La ecuatorianidad como identidad colectiva se revaloriza y se convierte en lo ecuatoriano. La interculturalidad está vinculada a conceptos como: diversidad, cultura, la lengua, políticas pocas integradoras en los estados, marcadas jerarquizaciones sociales, sistemas económicos excluyentes.
Es importante, remarcar que la interculturalidad no está limitada solo al ámbito local, regional porque transciende las fronteras. Cada inmigrante trae consigo su cultura y experiencias regionales del país o región de origen y experiencias migratorias internacionales. Estas experiencias que se expresan en lo simbólico, están cargadas de la cultura ancestral que enriquecen el contenido de la carrera de Educación Básica.

Un aspecto relevante de los diversos estudios interculturales es aplicado en el ámbito de la educación y los estudios del aprendizaje. La interculturalidad del siglo XXI tiene referentes sobre los modelos de comunicación de masas en Ecuador, los modelos de comunicación intercultural y de emigración. Los antropólogos Orozco, L. E. (2014), Pérez, M. L., y Argueta, A (2011) consideran que la interculturalidad expresa la interacción comunicativa que se produce entre dos o más diferentes culturas.

A los grupos en interacción mutua que se les denomina etnias, grupos sociales, grupos religiosos, culturas o comunidades es una denominación de preferencias de escuelas de las ciencias sociales y no de diferencias epistemológicas. Algunos estudios han venido definiendo la interculturalidad como la integración de culturas y la globalización como el proceso de destrucción de las identidades culturales. Es una afirmación, que pierde sentido en el contraste de los procesos interculturales. La interculturalidad es en esencia el proceso de compartir experiencias con otras culturas.

En este proceso se incorporan los aspectos positivos o no positivos de la cultura o identidad. Este un proceso que se realiza en la convivencia cultural. La Globalización como fenómeno mundial 
ha servido de motor de la movilidad de culturas a niveles globalizadores. El internet y los medios de comunicación han servido de comunicadores de intercambios culturales (Vinagre, $M$, 2014).

Como puede apreciarse la interculturalidad ocupa un lugar central en lo que a la formación del profesional de la carrera de Licenciatura en Educación Básica refiere, es por ello que, en el nuevo rediseño curricular propuesto, desde la multiculturalidad, las que contribuyen a la formación multicultural en los estudiantes de la carrera. Potenciándose así su competencia profesional y eficiencia en el desempeño laboral en los diferentes ámbitos de su accionar profesional.

En el caso específico de la carrera de Licenciatura de Educación Básica en la Facultad de Filosofía, Letra y Ciencias de la Educación, se realizan un conjunto de actividades docentes desde la multiculturalidad e interculturalidad, donde se programan ferias de exposición de conocimientos en las diferentes localidades de la región y del país, se exponen las canciones, exhibiciones gastronómicas, bailes, teatro del país y de otras regiones de América.

Actividades como pregones, casas abiertas y exposiciones culturales de diferente índole reflejan tradiciones y cultura de la región, muestra de las diferentes tradiciones de la región, investigaciones científicas, publicaciones de libros, artículos científicos, presentación de los resultados en eventos científicos nacionales e internacionales son las múltiples manifestaciones que tiene esta región de América para sus estudiantes universitarios de la carrera objeto de estudio.

Los aspectos antes señalados no son suficientes para el desarrollo de la concepción multicultural en la carrera, actualmente se está rediseñando la carrera en función de la enseñanza multicultural, donde se incluyen asignaturas que favorecen la educación multicultural, y la introducción metodológica de contenidos, actividades autónomas e investigativas, que permiten el desarrollo de competencias multiculturales, esto incluye incorporar la diversidad cultural en la evaluación de los estudiantes, procesos y sistema de gestión universitaria.

\section{Reflexiones finales}

La multiculturalidad es un reflejo de la diversidad socioeconómica, de formas de comercio, de mercadeo, psicología social, aspectos etnográficos, educación, en general, de la producción de bienes materiales y espirituales de que dispone la sociedad, que se evidencia, en sus costumbres, tradiciones y cultura en general, expresión de su identidad cultural, que se refleja en todos los aspectos del proceso de enseñanza universitaria.

La carrera de Educación Básica con un enfoque multicultural permitirá formar hombres con un alto nivel de socialización se adentra en el universo de la multiculturalidad, interculturalidad y transculturalidad, aspecto éste que se pone de manifiesto en la formación de un profesional en Educación Básica, altamente competente capaz de interactuar en los diferentes escenarios multiculturales. Es por ello que se fundamenta la necesidad de un rediseño curricular que desarrolle competencias vinculadas a la multiculturalidad, que incluya asignaturas tales como: Historia, Filosofía, Etnología, Etnografía, Antropología, Sociología entre otras, 
aplicadas a la enseñanza de este fenómeno de la multiculturalidad en la formación profesional del estudiante de Licenciatura en Educación Básica.

\section{Referencias bibliográficas}

Bensasson, Laura (2013), Educación intercultural en México ¿por qué y para quién?, Bruno, Baronnet y Medardo, Tapia (coords.). Educación e interculturali-dad, Política y políticas, México: UNAM, Recuperado de: http:// www. libros. UNAM. mx/digital, 7.

Castillo, Elizabeth, y Guido, Guido, Sandra (2015), La interculturalidad: ¿Principio o fin de la utopía?. Revista Colombiana de Educación, 69, pp.17-44. Recuperado de http:// revistas.pedagogica.edu.co/index.php/ RCE/article/ view/3244/2809

Clouet, Richard (2013), Understanding and assessing intercultural competence in an online environment: a case study of transnational education programme delivery between college students in ULPGC, Spain, and ICES, France. RESLA, 26, pp.139-157.

Cruz, Edwin (2013), Estado plurinacional, interculturalidad y autonomía indígena: Una reflexión sobre los casos de Bolivia y Ecuador. Revista Via iuris,14,pp.55-71. Recuperadodehttp://www.redalyc.org/ pdf/2739/273929754005.pdf

Dietz, Gunther, y Mateos, Laura (2011), Interculturalidad y educación intercultural en México. Un análisis de los discursos nacionales e internacionales en su impacto en los modelos educativos mexicanos. México: cgeib- sep, Recuperado dehttp://www.interculturalidadygenerocolsan.com.mx / v4 / pdf / GUNTHER \% 20 DIETZ .pdf

Gobierno Autónomo Descentralizado Municipal del Cantón Sucre (GAD) (2012), Plan de Desarrollo y Ordenamiento Territorial 2012- 2025, p.15-211.

Guber, Rosana (2001), La etnografía. Método, campo y reflexividad. Editorial Norma, Colombia.

Nocedo, Irma (1983), Metodología de la investigación Pedagógica y Psicológica. Editorial Pueblo y Educación. Ciudad de la Habana, pp. 18-88.

Olabuénaga, Ruiz, José (2012). Metodología de la investigación cualitativa (5a. ed.): Publicaciones de la Universidad de Deusto, pp. 45-67

Orozco, López, Efrén (2014), La Organización Sociedad Civil "Las Abejas". Procesos de lucha, resistencia y educación indígena (Tesis doctoral). Cesmeca-unicach, México.

Pérez, Maya, Lorena, y Argueta, Arturo (2011), Saberes indígenas y diálogo intercultural. Cultura y representaciones sociales. Revista electrónica de ciencias sociales, 5(10), pp.31-56.

Vinagre, Margarita (2014), El desarrollo de la competencia intercultural en los intercambios telecolaborativos, RED. Revista de Educación a Distancia, 13(41), pp.1-22.

- Esta obra está bajo una licencia de Creative Commons Reconocimiento-NoComercial-

Compartirlgual 3.0 Unported.

http://creativecommons.org/licenses/by-nc-sa/3.0/deed.es_ES 